\title{
Interpolation Problem for Stationary Sequences with Missing Observations
}

\author{
Mikhail Moklyachuk*, Maria Sidei \\ Department of Probability Theory, Statistics and Actuarial Mathematics,Taras Shevchenko National University of Kyiv, Ukraine
}

Received: 4 July 2015; Accepted: 16 July 2015

Editor: David G. Yu

Abstract The problem of the mean-square optimal estimation of the linear functional $A_{s} \xi=\sum_{l=0}^{s-1} \sum_{j=M_{l}}^{M_{l}+N_{l+1}} a(j) \xi(j)$, $M_{l}=\sum_{k=0}^{l}\left(N_{k}+K_{k}\right), \quad N_{0}=K_{0}=0$, which depends on the unknown values of a stochastic stationary sequence $\xi(j)$, $j \in \mathbb{Z}$ from observations of the sequence at points of time $j \in \mathbb{Z} \backslash S, S=\bigcup_{l=0}^{s-1}\left\{M_{l}, M_{l}+1, \ldots, M_{l}+N_{l+1}\right\}$ is considered. Formulas for calculating the mean-square error and the spectral characteristic of the optimal linear estimate of the functional are derived under the condition of spectral certainty, where the spectral density of the sequence $\xi(j)$ is exactly known. The minimax (robust) method of estimation is applied in the case where the spectral density is not known exactly, but sets of admissible spectral densities are given. Formulas that determine the least favourable spectral densities and the minimax spectral characteristics are derived for some special sets of admissible densities.

Keywords Stationary sequence, mean square error, minimax-robust estimate, least favourable spectral density, minimax spectral characteristic

AMS 2010 subject classifications. Primary: 60G10, 60G25, 60G35, Secondary: 62M20, 93E10, 93E11

DOI: $10.19139 /$ soic.v3i3.149

\section{Introduction}

The problem of estimation of the unknown values of stochastic processes is of constant interest in the theory of stochastic processes. The formulation of the interpolation, extrapolation and filtering problems for stationary stochastic sequences with known spectral densities and reducing them to the corresponding problems of the theory of functions belongs to A. N. Kolmogorov [18]. Effective methods of solution of the estimation problems for stationary stochastic sequences and processes were developed by N. Wiener [37] and A. M. Yaglom [38, 39]. Further results are presented in the books by Yu. A. Rozanov [34] and E. J. Hannan [13]. The crucial assumption of most of the methods developed for estimating the unobserved values of stochastic processes is that the spectral densities of the involved stochastic processes are exactly known. However, in practice complete information on the spectral densities is impossible in most cases. In this situation one finds parametric or nonparametric estimate of the unknown spectral density and then apply one of the traditional estimation methods provided that the selected density is the true one. This procedure can result in significant increasing of the value of error as K. S. Vastola and H. V. Poor [36] have demonstrated with the help of some examples. To avoid this effect one can search the estimates

*Correspondence to: Mikhail Moklyachuk (Email: Moklyachuk@gmail.com).

Department of Probability Theory, Statistics and Actuarial Mathematics, Taras Shevchenko National University of Kyiv, Volodymyrska 64 Str., Kyiv 01601, Ukraine.

ISSN 2310-5070 (online) ISSN 2311-004X (print)

Copyright (C) 2015 International Academic Press 
which are optimal for all densities from a certain class of admissible spectral densities. These estimates are called minimax since they minimize the maximum value of the error. The paper by Ulf Grenander [12] was the first one where this approach to extrapolation problem for stationary processes was proposed. Several models of spectral uncertainty and minimax-robust methods of data processing can be found in the survey paper by S. A. Kassam and H. V. Poor [17]. J. Franke [8], J. Franke and H. V. Poor [9] investigated the minimax extrapolation and filtering problems for stationary sequences with the help of convex optimization methods. This approach makes it possible to find equations that determine the least favorable spectral densities for different classes of densities. In the papers by M. P. Moklyachuk [26] - [29] the problems of extrapolation, interpolation and filtering for functionals which depend on the unknown values of stationary processes and sequences are investigated. The estimation problems for functionals which depend on the unknown values of multivariate stationary stochastic processes is the aim of the book by M. Moklyachuk and O. Masytka [30]. I. I. Dubovets'ka, O.Yu. Masyutka and M.P. Moklyachuk[3], I. I. Dubovets'ka and M. P. Moklyachuk [4] - [7], I. I. Golichenko and M. P. Moklyachuk [11] investigate the interpolation, extrapolation and filtering problems for periodically correlated stochastic sequences. The paper by M. M. Luz and M. P. Moklyachuk [20] - [24] deals with the estimation problems for functionals which depend on the unknown values of stochastic sequences with stationary increments. Prediction of stationary processes with missing observations is investigated in papers by P. Bondon [1, 2], Y. Kasahara, M. Pourahmadi and A. Inoue $[16,31]$.

In this article we consider the problem of the mean-square optimal estimation of the linear functional $A_{s} \xi=\sum_{l=0}^{s-1} \sum_{j=M_{l}}^{M_{l}+N_{l+1}} a(j) \xi(j), M_{l}=\sum_{k=0}^{l}\left(N_{k}+K_{k}\right), \quad N_{0}=K_{0}=0$, which depends on the unknown values of a stochastic stationary sequence $\xi(j), j \in \mathbb{Z}$, from observations of the sequence at points of time $j \in \mathbb{Z} \backslash S$, where $S=\bigcup_{l=0}^{s-1}\left\{M_{l}, M_{l}+1, \ldots, M_{l}+N_{l+1}\right\}$. The problem is investigated in the case of spectral certainty, where the spectral density is exactly known, and in the case of spectral uncertainty, where the spectral density is unknown, but a class of admissible spectral densities is given.

\section{The classical Hilbert space projection method of linear interpolation}

Let $\xi(j), j \in \mathbb{Z}$, be a (wide sense) stationary stochastic sequence. We will consider $\xi(j)$ as elements of the Hilbert space $H=L_{2}(\Omega, \mathcal{F}, P)$ of complex valued random variables with zero first moment, $E \xi=0$, finite second moment, $\underline{E|\xi|^{2}}<\infty$, and the inner product $(\xi, \eta)=E \xi \bar{\eta}$. The correlation function $R(k)=(\xi(j+k), \xi(j))=$ $E \xi(j+k) \overline{\xi(j)}$ of the stationary stochastic sequence $\xi(j), j \in \mathbb{Z}$, admits the spectral decomposition [10]

$$
R(k)=\int_{-\pi}^{\pi} e^{i k \lambda} F(d \lambda),
$$

where $F(d \lambda)$ is the spectral measure of the sequence. We will consider stationary stochastic sequences with absolutely continuous spectral measures and the correlation functions of the form

$$
R(k)=\frac{1}{2 \pi} \int_{-\pi}^{\pi} e^{i k \lambda} f(\lambda) d \lambda,
$$

where $f(\lambda)$ is the spectral density function of the sequence $\xi(j)$ that satisfies the minimality condition

$$
\int_{-\pi}^{\pi} f^{-1}(\lambda) d \lambda<\infty .
$$

This condition is necessary and sufficient in order that the error-free interpolation of unknown values of the sequence is impossible [34]. 
The stationary stochastic sequence $\xi(j), j \in \mathbb{Z}$, admits the spectral decomposition $[10,15]$

$$
\xi(j)=\int_{-\pi}^{\pi} e^{i j \lambda} Z(d \lambda)
$$

where $Z(\Delta)$ is the orthogonal stochastic measure of the sequence such that

$$
E Z\left(\Delta_{1}\right) \overline{Z\left(\Delta_{2}\right)}=F\left(\Delta_{1} \cap \Delta_{2}\right)=\frac{1}{2 \pi} \int_{\Delta_{1} \cap \Delta_{2}} f(\lambda) d \lambda
$$

Consider the problem of the mean-square optimal estimation of the linear functional

$$
A_{s} \xi=\sum_{l=0}^{s-1} \sum_{j=M_{l}}^{M_{l}+N_{l+1}} a(j) \xi(j), M_{l}=\sum_{k=0}^{l}\left(N_{k}+K_{k}\right), N_{0}=K_{0}=0,
$$

which depends on the unknown values of a stochastic stationary sequence $\xi(j), j \in \mathbb{Z}$, from observations of the sequence at points of time $j \in \mathbb{Z} \backslash S$, where $S=\bigcup_{l=0}^{s-1}\left\{M_{l}, M_{l}+1, \ldots, M_{l}+N_{l+1}\right\}$.

It follows from the spectral decomposition (2) of the sequence $\xi(j)$ that we can represent the functional $A_{s} \xi$ in the following form

$$
A_{s} \xi=\int_{-\pi}^{\pi} A_{s}\left(e^{i \lambda}\right) Z(d \lambda)
$$

where

$$
A_{s}\left(e^{i \lambda}\right)=\sum_{l=0}^{s-1} \sum_{j=M_{l}}^{M_{l}+N_{l+1}} a(j) e^{i j \lambda} .
$$

Denote by $H^{s}(\xi)$ the subspace of the Hilbert space $H=L_{2}(\Omega, \mathcal{F}, P)$ generated by elements $\{\xi(j): j \in \mathbb{Z} \backslash S\}$. Let $L_{2}(f)$ be the Hilbert space of complex-valued functions that are square-integrable with respect to the measure whose density is $f(\lambda)$. Denote by $L_{2}^{s}(f)$ the subspace of $L_{2}(f)$ generated by functions $\left\{e^{i j \lambda}, j \in \mathbb{Z} \backslash S\right\}$. The mean square optimal linear estimate $\hat{A}_{s} \xi$ of the functional $A_{s} \xi$ from observations of the sequence $\xi(j)$ at points of time $j \in \mathbb{Z} \backslash S$ is an element of the $H^{s}(\xi)$. It can be represented in the form

$$
\hat{A}_{s} \xi=\int_{-\pi}^{\pi} h\left(e^{i \lambda}\right) Z(d \lambda)
$$

where $h\left(e^{i \lambda}\right) \in L_{2}^{s}(f)$ is the spectral characteristic of the estimate $\hat{A}_{s} \xi$.

The mean square error $\Delta(h ; f)$ of the estimate $\hat{A}_{s} \xi$ is given by the formula

$$
\Delta(h ; f)=E\left|A_{s} \xi-\hat{A}_{s} \xi\right|^{2}=\frac{1}{2 \pi} \int_{-\pi}^{\pi}\left|A_{s}\left(e^{i \lambda}\right)-h\left(e^{i \lambda}\right)\right|^{2} f(\lambda) d \lambda .
$$

The Hilbert space projection method proposed by A. N. Kolmogorov [18] makes it possible to find the spectral characteristic $h\left(e^{i \lambda}\right)$ and the mean square error $\Delta(h ; f)$ of the optimal linear estimate of the functional $A_{s} \xi$ in the case where the spectral density $f(\lambda)$ of the sequence is exactly known and the minimality condition (1) is satisfied. The spectral characteristic can be found from the following conditions:

$$
\begin{aligned}
& \text { 1) } h\left(e^{i \lambda}\right) \in L_{2}^{s}(f), \\
& \text { 2) } A_{s}\left(e^{i \lambda}\right)-h\left(e^{i \lambda}\right) \perp L_{2}^{s}(f) .
\end{aligned}
$$


It follows from the second condition that for any $\eta \in H^{s}(\xi)$ the following equations should be satisfied

$$
\left(A_{s} \xi-\hat{A}_{s} \xi, \eta\right)=E\left(A_{s} \xi-\hat{A}_{s} \xi\right) \bar{\eta}=0
$$

The last relation is equivalent to equations

$$
E\left(A_{s} \xi-\hat{A}_{s} \xi\right) \bar{\xi}_{k}=0, \quad k \in \mathbb{Z} \backslash S .
$$

By using representations (3), (4) and definition of the inner product in $H$ we get

$$
\begin{aligned}
& E\left(\int_{-\pi}^{\pi}\left(A_{s}\left(e^{i \lambda}\right)-h\left(e^{i \lambda}\right)\right) Z(d \lambda) \cdot \int_{-\pi}^{\pi} e^{-i k \lambda} \overline{Z(d \lambda)}\right)= \\
& =\int_{-\pi}^{\pi}\left(A_{s}\left(e^{i \lambda}\right)-h\left(e^{i \lambda}\right)\right) f(\lambda) e^{-i k \lambda} d \lambda=0, k \in \mathbb{Z} \backslash S .
\end{aligned}
$$

It follows from this condition that the function $\left(A_{s}\left(e^{i \lambda}\right)-h\left(e^{i \lambda}\right)\right) f(\lambda)$ is of the form

$$
\begin{gathered}
\left(A_{s}\left(e^{i \lambda}\right)-h\left(e^{i \lambda}\right)\right) f(\lambda)=C_{s}\left(e^{i \lambda}\right), \\
C_{s}\left(e^{i \lambda}\right)=\sum_{l=0}^{s-1} \sum_{j=M_{l}}^{M_{l}+N_{l+1}} c(j) e^{i j \lambda},
\end{gathered}
$$

where $c(j), j \in S$ are unknown coefficients that we have to find.

From the last relation we deduce that the spectral characteristic $h\left(e^{i \lambda}\right)$ of the optimal linear estimate of the functional $A_{s} \xi$ is of the form

$$
h\left(e^{i \lambda}\right)=A_{s}\left(e^{i \lambda}\right)-C_{s}\left(e^{i \lambda}\right) f^{-1}(\lambda) .
$$

To find equations for determination the unknown coefficients $c(j), j \in S$, we use the decomposition of the function $f^{-1}(\lambda)$ into the Fourier series

$$
f^{-1}(\lambda)=\sum_{m=-\infty}^{\infty} r_{m} e^{i m \lambda}
$$

where $r_{m}$ are the Fourier coefficients of the function $f^{-1}(\lambda)$.

Inserting (6) into (5) we obtain the following representation of the spectral characteristic

$$
h\left(e^{i \lambda}\right)=\left(\sum_{l=0}^{s-1} \sum_{j=M_{l}}^{M_{l}+N_{l+1}} a(j) e^{i j \lambda}\right)-\left(\sum_{l=0}^{s-1} \sum_{j=M_{l}}^{M_{l}+N_{l+1}} c(j) e^{i j \lambda}\right)\left(\sum_{m=-\infty}^{\infty} r_{m} e^{i m \lambda}\right) .
$$

It follows from the first condition, $h\left(e^{i \lambda}\right) \in L_{2}^{s}(f)$, that the Fourier coefficients of the function $h\left(e^{i \lambda}\right)$ are equal to zero for $j \in S$, namely

$$
\int_{-\pi}^{\pi} h\left(e^{i \lambda}\right) e^{-i j \lambda} d \lambda=0, j \in S
$$


Using the last relations and (7) we get the following system of equations that determine the unknown coefficients $c(j), j \in S$,

$$
\begin{gathered}
a\left(M_{k-1}\right)-\sum_{l=0}^{s-1} \sum_{j=M_{l}}^{M_{l}+N_{l+1}} c(j) r_{M_{k-1}-j}=0 ; \\
a\left(M_{k-1}+1\right)-\sum_{l=0}^{s-1} \sum_{j=M_{l}}^{M_{l}+N_{l+1}} c(j) r_{M_{k-1}+1-j}=0 ; \\
\ldots \\
a\left(M_{k-1}+N_{k}\right)-\sum_{l=0}^{s-1} \sum_{j=M_{l}}^{M_{l}+N_{l+1}} c(j) r_{M_{k-1}+N_{k}-j}=0,
\end{gathered}
$$

where $k=1, \ldots, s$.

Denote by $\overrightarrow{\mathbf{a}}_{s}=\left(\vec{a}_{1}, \vec{a}_{2}, \ldots, \vec{a}_{s}\right), \vec{a}_{k}=\left(a\left(M_{k-1}\right), \ldots, a\left(M_{k-1}+N_{k}\right)\right), k=1, \ldots, s, q=N_{1}+N_{2}+\ldots+$ $N_{s}+s$. Let $B_{s}$ be a $q \times q$ matrix

$$
B_{s}=\left(\begin{array}{cccc}
B_{11} & B_{12} & \ldots & B_{1 s} \\
B_{21} & B_{22} & \ldots & B_{2 s} \\
\vdots & \vdots & \ddots & \vdots \\
B_{s 1} & B_{s 2} & \ldots & B_{s s}
\end{array}\right)
$$

where $B_{m n}$ are $\left(N_{m}+1\right) \times\left(N_{n}+1\right)$ matrices with elements that are Fourier coefficients of the function $f^{-1}(\lambda)$ :

$$
\begin{gathered}
B_{m n}(k, j)=\frac{1}{2 \pi} \int_{-\pi}^{\pi} f^{-1}(\lambda) e^{-i(k-j) \lambda} d \lambda=r_{k-j}, \\
k=M_{m-1}, \ldots, M_{m-1}+N_{m} \\
j=M_{n-1}, \ldots, M_{n-1}+N_{n} \\
m, n=1, \ldots, s
\end{gathered}
$$

Making use the introduced notations we can write formulas (8) in the form of equation

$$
\overrightarrow{\mathbf{a}}_{s}=B_{s} \overrightarrow{\mathbf{c}}_{s}
$$

where $\overrightarrow{\mathbf{c}}_{s}=\left(\vec{c}_{1}, \vec{c}_{2}, \ldots, \vec{c}_{s}\right), \vec{c}_{k}=\left(c\left(M_{k-1}\right), \ldots, c\left(M_{k-1}+N_{k}\right)\right), k=1, \ldots, s$, is a vector constructed from the unknown coefficients $c(j), j \in S$. Since the matrix $B_{s}$ is reversible [31], we get the formula

$$
\overrightarrow{\mathbf{c}}_{s}=B_{s}^{-1} \overrightarrow{\mathbf{a}}_{s} .
$$

Hence, the unknown coefficients $c(j), j \in S$, are calculated by the formula

$$
c(j)=\left(B_{s}^{-1} \overrightarrow{\mathbf{a}}_{s}\right)_{j},
$$

where $\left(B_{s}^{-1} \overrightarrow{\mathbf{a}}_{s}\right)_{j}$ is the $j$ component of the vector $B_{s}^{-1} \overrightarrow{\mathbf{a}}_{s}$, and the formula for calculating the spectral characteristic of the estimate $\hat{A}_{s} \xi$ is of the form

$$
h\left(e^{i \lambda}\right)=\left(\sum_{l=0}^{s-1} \sum_{j=M_{l}}^{M_{l}+N_{l+1}} a(j) e^{i j \lambda}\right)-\left(\sum_{l=0}^{s-1} \sum_{j=M_{l}}^{M_{l}+N_{l+1}}\left(B_{s}^{-1} \overrightarrow{\mathbf{a}}_{s}\right)_{j} e^{i j \lambda}\right)\left(\sum_{m=-\infty}^{\infty} r_{m} e^{i m \lambda}\right) .
$$


The mean square error of the estimate of the function can be calculated by the formula

$$
\begin{aligned}
\Delta(h ; f) & =\frac{1}{2 \pi} \int_{-\pi}^{\pi}\left|C_{s}\left(e^{i \lambda}\right)\right|^{2} f^{-1}(\lambda) d \lambda= \\
& =\int_{-\pi}^{\pi}\left(\sum_{l=0}^{s-1} \sum_{k=M_{l}}^{M_{l}+N_{l+1}} c(k) e^{i k \lambda}\right)\left(\sum_{l=0}^{s-1} \sum_{j=M_{l}}^{M_{l}+N_{l+1}} \overline{c(j)} e^{-i j \lambda}\right)\left(\sum_{m=-\infty}^{\infty} r_{m} e^{i m \lambda}\right) d \lambda= \\
& =\left\langle\overrightarrow{\mathbf{c}}_{s}, \overrightarrow{\mathbf{c}}_{s} B_{s}\right\rangle=\left\langle B_{s}^{-1} \overrightarrow{\mathbf{a}}_{s}, \overrightarrow{\mathbf{a}}_{s}\right\rangle,
\end{aligned}
$$

where $\langle\cdot, \cdot\rangle$ is the inner product in $C^{q}$.

Let us summarize our results and present them in the form of a theorem.

Theorem 2.1

Let $\xi(j)$ be a stationary stochastic sequence with the spectral density $f(\lambda)$ that satisfies the minimality condition (1). The mean square error $\Delta(h, f)$ and the spectral characteristic $h\left(e^{i \lambda}\right)$ of the optimal linear estimate $\hat{A}_{s} \xi$ of the functional $A_{s} \xi$ from observations of the sequence $\xi(j)$ at points of time $j \in \mathbb{Z} \backslash S$, where $S=\bigcup_{l=0}^{s-1}\left\{M_{l}, \ldots, M_{l}+\right.$ $\left.N_{l+1}\right\}$, can be calculated by formulas (12), (11).

Example 1. Consider the problem of linear interpolation of the functional $A_{2} \xi=a(0) \xi(0)+a(1) \xi(1)+a(5) \xi(5)$ which depends on the unknown values $\xi(0), \xi(1), \xi(5)$ of the stochastic sequence $\xi(j)$ from observations at points $j \in \mathbb{Z} \backslash S$, where $S=\{0,1\} \cup\{5\}$. In this case the spectral characteristic (7) of the estimate $\hat{A}_{2} \xi$ can be calculated by the formula

$$
h\left(e^{i \lambda}\right)=\left(a(0)+a(1) e^{i \lambda}+a(5) e^{5 i \lambda}\right)-\left(c(0)+c(1) e^{i \lambda}+c(5) e^{5 i \lambda}\right) \cdot f^{-1}(\lambda),
$$

where $f(\lambda)$ is a known spectral density, the function $f^{-1}(\lambda)$ admits the decomposition $f^{-1}(\lambda)=\sum_{m=-\infty}^{\infty} r_{m} e^{i \lambda m}$, and coefficients $c(0), c(1), c(5)$ are determined by the system of equations

$$
\begin{gathered}
a(0)=c(0) r_{0}+c(1) r_{-1}+c(5) r_{-5}, \\
a(1)=c(0) r_{1}+c(1) r_{0}+c(5) r_{-4}, \\
a(5)=c(0) r_{5}+c(1) r_{4}+c(5) r_{0} .
\end{gathered}
$$

The matrix $B_{2}$ is of the form

$$
\begin{gathered}
B_{2}=\left(\begin{array}{ll}
B_{11} & B_{12} \\
B_{21} & B_{22}
\end{array}\right), \\
B_{11}=\left(\begin{array}{cc}
r_{0} & r_{-1} \\
r_{1} & r_{0}
\end{array}\right), B_{12}=\left(\begin{array}{c}
r_{-5} \\
r_{-4}
\end{array}\right), B_{21}=\left(\begin{array}{ll}
r_{5} & r_{4}
\end{array}\right), B_{22}=\left(\begin{array}{l}
r_{0}
\end{array}\right) .
\end{gathered}
$$

Let $\overrightarrow{\mathbf{a}}_{2}=\left(\vec{a}_{1}, \vec{a}_{2}\right)$, where $\vec{a}_{1}=(a(0), a(1)), \vec{a}_{2}=(a(5))$, and let $\overrightarrow{\mathbf{c}}_{2}=\left(\vec{c}_{1}, \vec{c}_{2}\right)$, where $\vec{c}_{1}=(c(0), c(1)), \vec{c}_{2}=$ $(c(5))$. In this case equations (9) and (10) can be rewritten as

Denote by

$$
\begin{gathered}
\overrightarrow{\mathbf{a}}_{2}=B_{2} \overrightarrow{\mathbf{c}}_{2}, \\
\overrightarrow{\mathbf{c}}_{2}=B_{2}^{-1} \overrightarrow{\mathbf{a}}_{2} .
\end{gathered}
$$

$$
D=\operatorname{det}\left(B_{2}\right)=r_{0}^{3}-r_{0} r_{-4} r_{4}-r_{1} r_{-1} r_{0}+r_{1} r_{-5} r_{4}+r_{5} r_{-1} r_{-4}-r_{5} r_{-5} r_{0} .
$$

We get the following formulas for calculating the coefficients $c(0), c(1), c(5)$

$$
c(0)=\left[\left(r_{0}^{2}-r_{-4} r_{4}\right) a(0)-\left(r_{-1} r_{0}-r_{-5} r_{4}\right) a(1)+\left(r_{-1} r_{4}-r_{-5} r_{0}\right) a(5)\right] \cdot D^{-1},
$$




$$
\begin{gathered}
c(1)=\left[\left(-r_{1} r_{0}+r_{-4} r_{5}\right) a(0)+\left(r_{0}^{2}-r_{5} r_{-5}\right) a(1)-\left(r_{0} r_{-4}-r_{-5} r_{1}\right) a(5)\right] \cdot D^{-1}, \\
c(5)=\left[\left(r_{1} r_{4}-r_{0} r_{5}\right) a(0)-\left(r_{0} r_{4}-r_{5} r_{-1}\right) a(1)+\left(r_{0}^{2}-r_{-1} r_{1}\right) a(5)\right] \cdot D^{-1} .
\end{gathered}
$$

Thus, the unknown coefficients $c(0), c(1), c(5)$ in (13) are determined.

The mean square error of the estimate is calculated by the formula

$$
\begin{aligned}
& \Delta(f)=<B_{2}^{-1} \overrightarrow{\mathbf{a}}_{2}, \overrightarrow{\mathbf{a}}_{2}>=D^{-1}\left[(a(0))^{2} r_{0}^{2}-(a(0))^{2} r_{-4} r_{4}-a(0) a(1) r_{-1} r_{0}+a(0) a(1) r_{-5} r_{4}\right. \\
& +a(0) a(5) r_{-1} r_{-4}-a(0) a(5) r_{-5} r_{0}-a(0) a(1) r_{0} r_{1}+a(0) a(1) r_{-4} r_{5}+(a(1))^{2} r_{0}^{2} \\
& -(a(1))^{2} r_{5} r_{-5}-a(1) a(5) r_{0} r_{-4}+a(1) a(5) r_{-5} r_{1}+a(0) a(5) r_{1} r_{4}-a(0) a(5) r_{0} r_{5} \\
& \left.-a(1) a(5) r_{0} r_{4}+a(1) a(5) r_{-1} r_{5}+(a(5))^{2} r_{0}^{2}-(a(5))^{2} r_{1} r_{-1}\right] .
\end{aligned}
$$

Consider this problem for the spectral density

$$
f(\lambda)=\frac{1}{\left|1-\alpha e^{-i \lambda}\right|^{2}},|\alpha|<1 .
$$

In this case we have

$$
f^{-1}(\lambda)=\left|1-\alpha e^{-i \lambda}\right|^{2}=\left(1+|\alpha|^{2}-\bar{\alpha} e^{i \lambda}-\alpha e^{-i \lambda}\right)
$$

and the Fourier coefficients of the function $f^{-1}(\lambda)$ are as follows

$$
r_{0}=\left(1+|\alpha|^{2}\right), r_{1}=-\bar{\alpha}, r_{-1}=-\alpha .
$$

The spectral characteristic is calculated by the formula

$$
\begin{array}{r}
h\left(e^{i \lambda}\right)=\left(a(0)+a(1) e^{i \lambda}+a(5) e^{5 i \lambda}\right)-\left(c(0)+c(1) e^{i \lambda}+c(5) e^{5 i \lambda}\right) \times \\
\left(1+|\alpha|^{2}-\bar{\alpha} e^{i \lambda}-\alpha e^{-i \lambda}\right),
\end{array}
$$

where the coefficients $c(0), c(1), c(5)$ are calculated by the formulas

$$
\begin{gathered}
c(0)=\left[\left(1+|\alpha|^{2}\right)^{2} a(0)+\left(\alpha\left(1+|\alpha|^{2}\right)\right) a(1)\right] \cdot D^{-1}, \\
c(1)=\left[\left(\bar{\alpha}\left(1+|\alpha|^{2}\right)\right) a(0)+\left(1+|\alpha|^{2}\right)^{2} a(1)\right] \cdot D^{-1}, \\
c(5)=\left[\left(\left(1+|\alpha|^{2}\right)^{2}-|\alpha|^{2}\right) a(5)\right] \cdot D^{-1},
\end{gathered}
$$

with $D=\left(1+|\alpha|^{2}\right)\left(1+|\alpha|^{2}+|\alpha|^{4}\right)$.

Inserting values of $c(0), c(1), c(5)$ into the formula for calculating the spectral characteristic we obtain that

$$
\begin{aligned}
h\left(e^{i \lambda}\right)= & {\left[\left(a(0) \alpha\left(1+|\alpha|^{2}\right)^{2}+a(1) \alpha^{2}\left(1+|\alpha|^{2}\right)\right) e^{-i \lambda}+\right.} \\
& +\left(a(0)(\bar{\alpha})^{2}\left(1+|\alpha|^{2}\right)+a(1) \bar{\alpha}\left(1+|\alpha|^{2}\right)^{2}\right) e^{2 i \lambda}+ \\
& \left.+a(5) \alpha\left(1+|\alpha|^{2}+|\alpha|^{4}\right) e^{4 i \lambda}+a(5) \bar{\alpha}\left(1+|\alpha|^{2}+|\alpha|^{4}\right) e^{6 i \lambda}\right] \times \\
& \left(\left(1+|\alpha|^{2}\right)\left(1+|\alpha|^{2}+|\alpha|^{4}\right)\right)^{-1} .
\end{aligned}
$$


The mean square error is of the form

$$
\begin{aligned}
& \Delta(f)=D^{-1}\left[(a(0))^{2}\left(1+|\alpha|^{2}\right)^{2}+a(0) a(1) \alpha\left(1+|\alpha|^{2}\right)+a(0) a(1)\left(1+|\alpha|^{2}\right) \bar{\alpha}\right. \\
& \left.+(a(1))^{2}\left(1+|\alpha|^{2}\right)^{2}+(a(5))^{2}\left(1+|\alpha|^{2}\right)^{2}-(a(5))^{2}|\alpha|^{2}\right] .
\end{aligned}
$$

a) Let $a(0)=1, a(1)=0, a(5)=1$. We get the following formula for calculating the spectral characteristic

$$
h\left(e^{i \lambda}\right)=\left(1+e^{5 i \lambda}\right)-\left(c(0)+c(1) e^{i \lambda}+c(5) e^{5 i \lambda}\right)\left(1+|\alpha|^{2}-\bar{\alpha} e^{i \lambda}-\alpha e^{-i \lambda}\right),
$$

and formulas for calculating coefficients $c(0), c(1), c(5)$

$$
\begin{gathered}
c(0)=\left(1+|\alpha|^{2}\right) /\left(1+|\alpha|^{2}+|\alpha|^{4}\right), \\
c(1)=\bar{\alpha} /\left(1+|\alpha|^{2}+|\alpha|^{4}\right) \\
c(5)=1 /\left(1+|\alpha|^{2}\right)
\end{gathered}
$$

Thus, the spectral characteristic of the estimate $\hat{A}_{2}$ is of the form

$$
\begin{aligned}
h\left(e^{i \lambda}\right)= & {\left[\alpha\left(1+|\alpha|^{2}\right)^{2} e^{-i \lambda}+(\bar{\alpha})^{2}\left(1+|\alpha|^{2}\right) e^{2 i \lambda}+\right.} \\
& \left.+\alpha\left(1+|\alpha|^{2}+|\alpha|^{4}\right) e^{4 i \lambda}+\bar{\alpha}\left(1+|\alpha|^{2}+|\alpha|^{4}\right) e^{6 i \lambda}\right] \times \\
& \left(\left(1+|\alpha|^{2}\right)\left(1+|\alpha|^{2}+|\alpha|^{4}\right)\right)^{-1} .
\end{aligned}
$$

The mean square error of the estimate $\hat{A}_{2}$ is calculated by the formula

$$
\Delta_{1}=\Delta(f)=\left(2\left(1+|\alpha|^{2}\right)^{2}-|\alpha|^{2}\right) /\left(1+|\alpha|^{2}\right)\left(1+|\alpha|^{2}+|\alpha|^{4}\right) .
$$

b) Let $a(0)=1, a(1)=0, a(5)=0$. We get that

$$
h\left(e^{i \lambda}\right)=1+\left(c(0)+c(1) e^{i \lambda}\right)\left(1+|\alpha|^{2}-\bar{\alpha} e^{i \lambda}-\alpha e^{-i \lambda}\right),
$$

where coefficients $c(0), c(1)$ are calculated by the formulas

$$
\begin{gathered}
c(0)=\left(1+|\alpha|^{2}\right) /\left(1+|\alpha|^{2}+|\alpha|^{4}\right), \\
c(1)=\bar{\alpha} /\left(1+|\alpha|^{2}+|\alpha|^{4}\right),
\end{gathered}
$$

and $c(5)=0$.

The spectral characteristic of the estimate $\hat{A}_{2} \xi$ is of the form

$$
h\left(e^{i \lambda}\right)=\left(\alpha\left(1+|\alpha|^{2}\right)^{2} e^{-i \lambda}+(\bar{\alpha})^{2}\left(1+|\alpha|^{2}\right) e^{2 i \lambda}\right)\left(1+|\alpha|^{2}+|\alpha|^{4}\right)^{-1} .
$$

The mean square error is calculated by the formula

$$
\Delta_{2}=\Delta(f)=\left(1+|\alpha|^{2}\right) /\left(1+|\alpha|^{2}+|\alpha|^{4}\right) .
$$

It is obvious that $\Delta_{1}>\Delta_{2}$. 
Example 2. Consider the problem of linear interpolation of the functional $A_{3} \xi=a(-n) \xi(-n)+a(0) \xi(0)+$ $a(m) \xi(m)$ which depends on the unknown values $\xi(-n), \xi(0), \xi(m)$ of the stochastic sequence $\xi(j)$ from observations of the sequence at points $j \in \mathbb{Z} \backslash S$, where $S=\{-n\} \cup\{0\} \cup\{m\}$.

The spectral characteristic of the estimate $\hat{A}_{3} \xi$ is of the form

$$
h\left(e^{i \lambda}\right)=\left(a(-n) e^{-n i \lambda}+a(0)+a(m) e^{m i \lambda}\right)-\left(c(-n) e^{-n i \lambda}+c(0)+c(m) e^{m i \lambda}\right) \cdot f^{-1}(\lambda),
$$

where $f^{-1}(\lambda)=\sum_{m=-\infty}^{\infty} r_{m} e^{i \lambda m}$, and coefficients $c(-n), c(0), c(m)$ are calculated by the formulas

$$
\begin{array}{r}
a(-n)=c(-n) r_{0}+c(0) r_{-n}+c(m) r_{-n-m}, \\
a(0)=c(-n) r_{n}+c(0) r_{0}+c(m) r_{-m}, \\
a(m)=c(-n) r_{n+m}+c(0) r_{m}+c(m) r_{0} .
\end{array}
$$

The matrix $B_{3}$ is of the form

$$
\begin{gathered}
B_{3}=\left(\begin{array}{lll}
B_{11} & B_{12} & B_{13} \\
B_{21} & B_{22} & B_{23} \\
B_{31} & B_{32} & B_{33}
\end{array}\right), \\
B_{11}=\left(r_{0}\right), B_{12}=\left(r_{-n}\right), B_{13}=\left(r_{-n-m}\right), \\
B_{21}=\left(r_{n}\right), B_{22}=\left(r_{0}\right), B_{23}=\left(r_{-m}\right), \\
B_{31}=\left(r_{n+m}\right), B_{32}=\left(r_{m}\right), B_{33}=\left(r_{0}\right) .
\end{gathered}
$$

Let $\overrightarrow{\mathbf{a}}_{3}=\left(\vec{a}_{1}, \vec{a}_{2}, \vec{a}_{3}\right)$, where $\vec{a}_{1}=(a(-n)), \vec{a}_{2}=(a(0)), \vec{a}_{3}=(a(m))$, and let $\overrightarrow{\mathbf{c}}_{3}=\left(\vec{c}_{1}, \vec{c}_{2}, \vec{c}_{3}\right)$, where $\vec{c}_{1}=$ $(c(-n)), \vec{c}_{2}=(c(0)), \vec{c}_{3}=(c(m))$.

In this case equations (9) and (10) can be written as

$$
\begin{gathered}
\overrightarrow{\mathbf{a}}_{3}=B_{3} \overrightarrow{\mathbf{c}}_{3}, \\
\overrightarrow{\mathbf{c}}_{3}=B_{3}^{-1} \overrightarrow{\mathbf{a}}_{3} .
\end{gathered}
$$

Denote by

$$
D=\operatorname{det}\left(B_{3}\right)=r_{0}^{3}-r_{0} r_{-m} r_{m}-r_{n} r_{-n} r_{0}+r_{n} r_{-n-m} r_{m}+r_{n+m} r_{-n} r_{-m}-r_{n+m} r_{-n-m} r_{0} .
$$

Then

$$
\begin{gathered}
c(-n)=\left[\left(r_{0}^{2}-r_{-m} r_{m}\right) a(-n)-\left(r_{-n} r_{0}-r_{-n-m} r_{m}\right) a(0)+\left(r_{-n} r_{m}-r_{-n-m} r_{0}\right) a(m)\right] / D, \\
c(0)=\left[\left(-r_{n} r_{0}+r_{-m} r_{n+m}\right) a(-n)+\left(r_{0}^{2}-r_{n+m} r_{-n-m}\right) a(0)-\left(r_{0} r_{-m}-r_{-n-m} r_{n}\right) a(m)\right] / D, \\
c(m)=\left[\left(r_{n} r_{m}-r_{0} r_{n+m}\right) a(-n)-\left(r_{0} r_{m}-r_{n+m} r_{-n}\right) a(0)+\left(r_{0}^{2}-r_{-n} r_{n}\right) a(m)\right] / D .
\end{gathered}
$$

The mean square error is calculated by the formula

$$
\begin{aligned}
& \Delta(f)=<B_{3}^{-1} \overrightarrow{\mathbf{a}}_{3}, \overrightarrow{\mathbf{a}}_{3}>=D^{-1}\left[(a(-n))^{2} r_{0}^{2}-(a(-n))^{2} r_{-m} r_{m}-a(-n) a(0) r_{-n} r_{0}\right. \\
& +a(-n) a(0) r_{-n-m} r_{m}+a(-n) a(m) r_{-n} r_{-m}-a(-n) a(m) r_{-n-m} r_{0}-a(-n) a(0) r_{0} r_{n} \\
& +a(-n) a(0) r_{-m} r_{n+m}+(a(0))^{2} r_{0}^{2}-(a(0))^{2} r_{n+m} r_{-n-m}-a(0) a(m) r_{0} r_{-m} \\
& +a(0) a(m) r_{-n-m} r_{n}+a(-n) a(m) r_{n} r_{m}-a(-n) a(m) r_{0} r_{n+m}-a(0) a(m) r_{0} r_{m} \\
& \left.+a(0) a(m) r_{-n} r_{n+m}+(a(m))^{2} r_{0}^{2}-(a(m))^{2} r_{n} r_{-n}\right] .
\end{aligned}
$$


Consider the problem of estimation of the functional $A_{3}(\xi)=\xi(-n)+\xi(0)+\xi(m)$ in the case where the spectral density of the stochastic sequence is of the form

$$
f(\lambda)=\frac{1}{\left|1-\alpha e^{-i \lambda}\right|^{2}},|\alpha|<1
$$

In this case $f^{-1}(\lambda)=\left(1+|\alpha|^{2}-\bar{\alpha} e^{i \lambda}-\alpha e^{-i \lambda}\right)$ and all Fourier coefficients of the function $f^{-1}(\lambda)$, except $r_{0}, r_{1}, r_{-1}$, are equal to zero 0 .

Consider the following four cases: 1$) n \neq 1, m \neq 1$; 2$) n \neq 1, m=1$; 3) $n=1, m \neq 1$; 4$) n=1, m=1$.

The spectral characteristic and the mean square error of the estimate $\hat{A}_{3} \xi$ of the functional $A_{3}(\xi)$ are calculated by the following formulas.

In the first case

$$
\begin{aligned}
& h\left(e^{i \lambda}\right)=\left(\alpha e^{(-n-1) i \lambda}+\bar{\alpha} e^{(-n+1) i \lambda}+\alpha e^{-i \lambda}+\bar{\alpha} e^{i \lambda}+\alpha e^{(m-1) i \lambda}+\right. \\
&\left.\bar{\alpha} e^{(m+1) i \lambda}\right)\left(1+|\alpha|^{2}\right)^{-2} \\
& \Delta(f)=3 /\left(1+|\alpha|^{2}\right)
\end{aligned}
$$

In the second case

$$
\begin{aligned}
h\left(e^{i \lambda}\right)= & \left(\alpha\left(1+|\alpha|^{2}\right)+\alpha^{2}\right)\left(1+|\alpha|^{2}\right) e^{-i \lambda}+ \\
& \left((\bar{\alpha})^{2}+\bar{\alpha}\left(1+|\alpha|^{2}\right)\right)\left(1+|\alpha|^{2}\right) e^{2 i \lambda}+ \\
& \left.\bar{\alpha}\left(1+|\alpha|^{2}+|\alpha|^{4}\right) e^{(-n+1) i \lambda}+\alpha\left(1+|\alpha|^{2}+|\alpha|^{4}\right) e^{(-n-1) i \lambda}\right] \times \\
& \left(\left(1+|\alpha|^{2}\right)\left(1+|\alpha|^{2}+|\alpha|^{4}\right)\right)^{-1}, \\
\Delta(f)= & \left(3\left(1+|\alpha|^{2}\right)-|\alpha|^{2}+\left(1+|\alpha|^{2}\right)(\alpha+\bar{\alpha})\right) . \\
& \left(\left(1+|\alpha|^{2}\right)\left(1+|\alpha|^{2}+|\alpha|^{4}\right)\right)^{-1} ;
\end{aligned}
$$

In the third case

$$
\begin{aligned}
h\left(e^{i \lambda}\right)= & {\left[(\bar{\alpha})^{2}+\bar{\alpha}\left(1+|\alpha|^{2}\right)\right)\left(1+|\alpha|^{2}\right) e^{i \lambda}+} \\
& \left(\alpha^{2}+\alpha\left(1+|\alpha|^{2}\right)\right)\left(1+|\alpha|^{2}\right) e^{-2 i \lambda}+ \\
& \left.\bar{\alpha}\left(1+|\alpha|^{2}+|\alpha|^{4}\right) e^{(m+1) i \lambda}+\alpha\left(1+|\alpha|^{2}+|\alpha|^{4}\right) e^{(m-1) i \lambda}\right] \times \\
& \left(\left(1+|\alpha|^{2}\right)\left(1+|\alpha|^{2}+|\alpha|^{4}\right)\right)^{-1}, \\
\Delta(f)= & \left(3\left(1+|\alpha|^{2}\right)-|\alpha|^{2}+\left(1+|\alpha|^{2}\right)(\alpha+\bar{\alpha})\right) . \\
& \left(\left(1+|\alpha|^{2}\right)\left(1+|\alpha|^{2}+|\alpha|^{4}\right)\right)^{-1} ;
\end{aligned}
$$


In the fourth case

$$
\begin{aligned}
h\left(e^{i \lambda}\right)= & {\left[\left((\bar{\alpha})^{2}+\bar{\alpha}\left(1+|\alpha|^{2}\right)+\left(1+|\alpha|^{2}+|\alpha|^{4}\right)\right) \bar{\alpha} e^{2 i \lambda}+\right.} \\
& \left.\left(\left(1+|\alpha|^{2}+|\alpha|^{4}\right)+\alpha\left(1+|\alpha|^{2}\right)+|\alpha|^{2}\right) \alpha e^{-2 i \lambda}\right] \times \\
& \left(1+|\alpha|^{2}+|\alpha|^{4}+|\alpha|^{6}\right)^{-1}, \\
\Delta(f)= & \left(3\left(1+|\alpha|^{2}\right)-|\alpha|^{2}+\left(1+|\alpha|^{2}\right)(\alpha+\bar{\alpha})+\left(\alpha^{2}+\bar{\alpha}^{2}\right)\right) . \\
& \left(\left(1+|\alpha|^{2}\right)\left(1+|\alpha|^{2}+|\alpha|^{4}\right)\right)^{-1} .
\end{aligned}
$$

\section{Minimax-robust method of interpolation}

The traditional methods of estimation of the functional $A_{s} \xi$ which depends on unknown values of a stationary stochastic sequence $\xi(j)$ can be applied in the case where the spectral density $f(\lambda)$ of the considered stochastic sequence $\xi(j)$ is exactly known. In practise, however, we do not have complete information on spectral density of the sequence. For this reason we apply the minimax(robust) method of estimation of the functional $A_{s} \xi$, that is we find an estimate that minimizes the maximum of the mean square errors for all spectral densities from the given class of admissible spectral densities $D$.

Definition 3.1. For a given class of spectral densities $D$ a spectral density $f_{0}(\lambda) \in D$ is called the least favourable in $D$ for the optimal linear estimation of the functional $A_{s} \xi$ if the following relation holds true

$$
\Delta\left(f_{0}\right)=\Delta\left(h\left(f_{0}\right) ; f_{0}\right)=\max _{f \in D} \Delta(h(f) ; f) .
$$

Definition 3.2. For a given class of spectral densities $D$ the spectral characteristic $h^{0}\left(e^{i \lambda}\right)$ of the optimal linear estimate of the functional $A_{s} \xi$ is called minimax-robust if

$$
\begin{gathered}
h^{0}\left(e^{i \lambda}\right) \in H_{D}=\bigcap_{f \in D} L_{2}^{s}(f), \\
\min _{h \in H_{D}} \max _{f \in D} \Delta(h ; f)=\sup _{f \in D} \Delta\left(h^{0} ; f\right) .
\end{gathered}
$$

It follows from the introduced definitions and the obtained formulas that the following statement holds true.

\section{Lemma 3.1}

The spectral density $f_{0}(\lambda) \in D$ is the least favourable in the class of admissible spectral densities $D$ for the optimal linear estimate of the functional $A_{s} \xi$ if the Fourier coefficients of the function $f_{0}^{-1}(\lambda)$ define a matrix $B_{s}^{0}$ that is a solution to the optimization problem

$$
\max _{f \in D}\left\langle B_{s}^{-1} \overrightarrow{\mathbf{a}}_{s}, \overrightarrow{\mathbf{a}}_{s}\right\rangle=\left\langle\left(B_{s}^{0}\right)^{-1} \overrightarrow{\mathbf{a}}_{s}, \overrightarrow{\mathbf{a}}_{s}\right\rangle .
$$

The minimax spectral characteristic $h^{0}=h\left(f_{0}\right)$ can be calculated by the formula (11) if $h\left(f_{0}\right) \in H_{D}$.

The least favourable spectral density $f_{0}$ and the minimax spectral characteristic $h^{0}$ form a saddle point of the function $\Delta(h ; f)$ on the set $H_{D} \times D$. The saddle point inequalities

$$
\Delta\left(h ; f_{0}\right) \geq \Delta\left(h^{0} ; f_{0}\right) \geq \Delta\left(h^{0} ; f\right) \quad \forall f \in D, \forall h \in H_{D}
$$

hold true if $h^{0}=h\left(f_{0}\right)$ and $h\left(f_{0}\right) \in H_{D}$, where $f_{0}$ is a solution to the constrained optimization problem

$$
\tilde{\Delta}(f)=-\Delta\left(h^{0} ; f\right)=-\frac{1}{2 \pi} \int_{-\pi}^{\pi} \frac{\left|C_{s}^{0}\left(e^{i \lambda}\right)\right|^{2}}{f_{0}^{2}(\lambda)} f(\lambda) d \lambda \rightarrow \inf , \quad f(\lambda) \in D
$$


where

$$
C_{s}^{0}\left(e^{i \lambda}\right)=\sum_{l=0}^{s-1} \sum_{j=M_{l}}^{M_{l}+N_{l+1}}\left(\left(B_{s}^{0}\right)^{-1} \overrightarrow{\mathbf{a}}_{s}\right)_{j} e^{i j \lambda} .
$$

The constrained optimization problem (15) is equivalent to the unconstrained optimization problem

$$
\Delta_{D}(f)=\tilde{\Delta}(f)+\delta(f \mid D) \rightarrow \inf ,
$$

where $\delta(f \mid D)$ is the indicator function of the set $D$. Solution $f_{0}$ to this problem is characterized by the condition $0 \in \partial \Delta_{D}\left(f_{0}\right)$, where $\partial \Delta_{D}\left(f_{0}\right)$ is the subdifferential of the convex functional $\Delta_{D}(f)$ at point $f_{0}$. This condition makes it possible to find the least favourable spectral densities in some special classes of spectral densities $D$ [14], [32], [33].

Note, that the form of the functional $\Delta\left(h^{0} ; f\right)$ is convenient for application the Lagrange method of indefinite multipliers for finding solution to the problem (15). Making use the method of Lagrange multipliers and the form of subdifferentials of the indicator functions we describe relations that determine least favourable spectral densities in some special classes of spectral densities (see books [11, 29, 30] for additional details).

\section{Least favourable spectral densities in the class $D_{0}^{-}$}

Consider the problem of the optimal estimation of the functional $A_{s} \xi$ which depends on the unknown values of a stationary stochastic sequence $\xi(j)$ in the case where the spectral density is from the class

$$
D_{0}^{-}=\left\{\begin{array}{l|l}
f(\lambda) & \frac{1}{2 \pi} \int_{-\pi}^{\pi} f^{-1}(\lambda) d \lambda \geq P
\end{array}\right\}
$$

Let the sequence $a(k), k \in S$, that determines the functional $A_{s} \xi$, be strictly positive. To find solutions to the constrained optimization problem (15) we use the Lagrange multipliers method. With the help of this method we get the equation

$$
\frac{1}{2 \pi} \int_{-\pi}^{\pi}\left[\frac{\left|C_{s}^{0}\left(e^{i \lambda}\right)\right|^{2}}{f_{0}^{2}(\lambda)}-p_{0}^{2} \frac{1}{f_{0}^{2}(\lambda)}\right] \rho(f(\lambda)) d \lambda=0,
$$

where $p_{0}^{2}$ is a constant (the Lagrange multiplier), $\rho(f(\lambda))$ is a variation of the function $f(\lambda)$. From a generalization of the Lagrange lemma we get that the Fourier coefficients of the function $f_{0}^{-1}$ satisfy the equation

$$
\left|\sum_{l=0}^{s-1} \sum_{k=M_{l}}^{M_{l}+N_{l+1}} c(k) e^{i k \lambda}\right|^{2}=p_{0}^{2},
$$

where $c(k), k \in S$, are components of the vector $\overrightarrow{\mathbf{c}}_{s}$ that satisfies the equation $B_{s}^{0} \overrightarrow{\mathbf{c}}_{s}=\overrightarrow{\mathbf{a}}_{s}$, the matrix $B_{s}^{0}$ consists from matrices $B_{m n}^{0}(k, j)$, each of which is determined by the Fourier coefficients of the function $f_{0}^{-1}(\lambda)$

$$
\begin{gathered}
B_{m n}^{0}(k, j)=\frac{1}{2 \pi} \int_{-\pi}^{\pi} f_{0}^{-1}(\lambda) e^{-i(k-j) \lambda} d \lambda=r_{k-j}^{0} \\
k=M_{m-1}, \ldots, M_{m-1}+N_{m} \\
j=M_{n-1}, \ldots, M_{n-1}+N_{n} \\
m, n=1, \ldots, s
\end{gathered}
$$


The Fourier coefficients $r_{k}=r_{-k}, k \in S$, satisfy both equation (16) and equation $B_{s}^{0} \overrightarrow{\mathbf{c}}_{s}=\overrightarrow{\mathbf{a}}_{s}$. These coefficients can be found from the equation $B_{s}^{0} \overrightarrow{\mathbf{p}}_{s}^{0}=\overrightarrow{\mathbf{a}}_{s}$, where $\overrightarrow{\mathbf{p}}_{s}^{0}=\left(p_{0}, 0, \ldots, 0\right)$. The last relation can be presented in the form of the system of equations

$$
r_{k} p_{0}=a(k), k \in S .
$$

From the first equation of the system (for $k=0$ ) we find the unknown value $p_{0}=a(0)\left(r_{0}\right)^{-1}$. It follows from the extremum condition (14) and the restriction on the spectral densities from the class $D_{0}^{-}$that the Fourier coefficient $r_{0}=\frac{1}{2 \pi} \int_{-\pi}^{\pi} f_{0}^{-1}(\lambda) d \lambda=P$. Thus,

$$
r_{k}=P a(k) a^{-1}(0)
$$

Let

$$
r_{k}=r_{-k}= \begin{cases}P a(k) a^{-1}(0) & \text { if } k \in S \\ 0 & \text { if } k \in\left\{0, \ldots, M_{s-1}+N_{s}\right\} \backslash S .\end{cases}
$$

We can represent the function $f_{0}^{-1}(\lambda)$ in the form

$$
f_{0}^{-1}(\lambda)=\sum_{k=-\left(M_{s-1}+N_{s}\right)}^{M_{s-1}+N_{s}} r_{k} e^{i k \lambda} .
$$

Since the sequence $a(k), k \in S$, is strictly positive, the sequence $r_{k}, k=0,1, \ldots, M_{s-1}+N_{s}$, is also strictly positive and the function $f_{0}^{-1}(\lambda)$ is positive, so it can be represented in the form [19]

$$
f_{0}^{-1}(\lambda)=\left|\sum_{k=0}^{M_{s-1}+N_{s}} \gamma_{k} e^{-i k \lambda}\right|^{2}, \lambda \in[-\pi, \pi],
$$

where $\gamma_{k}=0, k \in\left\{0, \ldots, M_{s-1}+N_{s}\right\} \backslash S$. Hence, $f_{0}(\lambda)$ is the spectral density of the autoregressive stochastic sequence of order $M_{s-1}+N_{s}$ generated by the equation

$$
\sum_{k=0}^{M_{s-1}+N_{s}} \gamma_{k} \xi(n-k)=\sum_{l=0}^{s-1} \sum_{k=M_{l}}^{M_{l}+N_{l+1}} \gamma_{k} \xi(n-k)=\epsilon_{n},
$$

where $\epsilon_{n}$ is a "white noise" sequence.

The minimax spectral characteristic $h\left(f_{0}\right)$ of the optimal linear estimate of the functional $A_{s} \xi$ can be calculated by the formula (5), where

$$
C_{N}\left(e^{i \lambda}\right)=\sum_{l=0}^{s-1} \sum_{k=M_{l}}^{M_{l}+N_{l+1}} c(k) e^{i k \lambda}=p_{0}=P^{-1} a(0),
$$

namely

$$
\begin{gathered}
h\left(f_{0}\right)=\sum_{l=0}^{s-1} \sum_{k=M_{l}}^{M_{l}+N_{l+1}} a(k) e^{i k \lambda}-P^{-1} a(0) \sum_{k=-\left(M_{s-1}+N_{s}\right)}^{M_{s-1}+N_{s}} r_{k} e^{i k \lambda} \\
=\sum_{k=1}^{N_{1}} a(k) e^{-i k \lambda}+\sum_{l=1}^{s-1} \sum_{k=M_{l}}^{M_{l}+N_{l+1}} a(k) e^{-i k \lambda} .
\end{gathered}
$$

Summing up our reasoning we come to conclusion that the following theorem holds true.

\section{Theorem 4.1}

The least favourable in the class $D_{0}^{-}$spectral density for the optimal linear estimation of the functional $A_{s} \xi$ determined by strictly positive sequence $a(k), k \in S$, is the spectral density of the autoregressive sequence (17) whose Fourier coefficients are $r_{k}=r_{-k}=P a(k) a^{-1}(0), k \in S$. The minimax spectral characteristics $h\left(f_{0}\right)$ is given by formula (18). 
Example 3. Consider the problem of the optimal linear estimation of the functional $A_{2} \xi=2 \xi(0)+\xi(2)$ which depends on the unknown values $\xi(0), \xi(2)$ of the stationary stochastic sequence $\{\xi(j): j \in \mathbb{Z}\}$ from observations of the sequence at points of time $\mathbb{Z} \backslash\{0,2\}$. The system of equations that determine the Fourier coefficients of the least favourable spectral density in the class $D_{0}^{-}$is of the form

$$
\begin{aligned}
& r_{0} p_{0}=2 ; \\
& r_{2} p_{0}=1 .
\end{aligned}
$$

We have $r_{0}=P, r_{2}=r_{-2}=\frac{1}{2} P$. The least favourable spectral density is of the form

$$
f_{0}=1 /\left|x+y e^{2 i \lambda}\right|
$$

where $x= \pm \sqrt{\frac{P}{2}}, y= \pm \sqrt{\frac{P}{2}}$. The minimax spectral characteristic can by calculated by the formula

$$
h\left(f_{0}\right)=-\sqrt{\frac{P}{2}} e^{-2 i \lambda} .
$$

\section{Least favourable spectral densities in the class $D_{W}$}

Consider the problem of the optimal estimation of the functional $A_{s} \xi$ which depends on the unknown values of a stationary stochastic sequence $\xi(j)$ in the case where the spectral density of the sequence is from the set of spectral densities with restrictions on the moments of the function $f^{-1}(\lambda)$. Let

$$
D_{W}=\left\{f(\lambda) \mid \frac{1}{2 \pi} \int_{-\pi}^{\pi} f^{-1}(\lambda) \cos (w \lambda) d \lambda=r_{w}, w=0,1, \ldots, W\right\},
$$

where $r_{w}, w=0,1, \ldots, W$ is a strictly positive sequence. There is an infinite number of functions in the class $D_{W}$ [19] and the function

$$
f^{-1}(\lambda)=\sum_{w=-W}^{W} r_{|w|} e^{i w \lambda}>0, \lambda \in[-\pi, \pi] .
$$

To find solutions to the constrained optimization problem (15) for the set $D_{W}$ of admissible spectral densities we use the Lagrange multipliers method and the equation

$$
\left|\sum_{l=0}^{s-1} \sum_{k=M_{l}}^{M_{l}+N_{l+1}} c(k) e^{i k \lambda}\right|^{2}=\sum_{w=0}^{W} \alpha_{w} \cos (w \lambda)=\left|\sum_{w=0}^{W} p(w) e^{i w \lambda}\right|^{2},
$$

where $\alpha_{w}, w=0,1, \ldots, W$ are the Lagrange multipliers and $c(k), k=0, \ldots, W$ are solutions to the equation $B_{s}^{0} \overrightarrow{\mathbf{c}}_{s}=\overrightarrow{\mathbf{a}}_{s}$.

Consider two cases: $W \geq M_{s-1}+N_{s}$ and $W<M_{s-1}+N_{s}$. Let $W \geq M_{s-1}+N_{s}$. In this case the given Fourier coefficients $r_{w}$ define the matrix $B_{s}^{0}$ and the optimization problem (14) is degenerate. Let $p\left(M_{s-1}+\right.$ $\left.N_{s}+1\right)=\ldots=p(W)=0$ and $p(j)=0, j \notin S$. Components $p(j), j \in S$, of the vector $\overrightarrow{\mathbf{p}}_{s}$ can be found from the equation $B_{s}^{0} \overrightarrow{\mathbf{p}}_{s}=\overrightarrow{\mathbf{a}}_{s}$. Hence, the relation (19) holds true. Thus the least favorable is every density $f(\lambda) \in D_{W}$ and the density of the autoregression stochastic sequence

$$
f_{0}(\lambda)=1 / \sum_{w=-W}^{W} r_{|w|} e^{i w \lambda}=1 /\left|\sum_{k=0}^{W} \gamma_{k} e^{i k \lambda}\right|
$$

is least favorable, too. 
Let $W<M_{s-1}+N_{s}$. Then the matrix $B_{s}$ is determined by the known $r_{w}, w \in S \cap\{0, \ldots, W\}$, and the unknown $r_{w}, w \in S \backslash\{0, \ldots, W\}$, Fourier coefficients of the function $f^{-1}(\lambda)$. The unknown coefficients $p(k)$, $k \in S \cap\{0, \ldots, W\}$, and $r_{w}, w \in S \backslash\{0, \ldots, W\}$, can be found from the equation $B_{s} \overrightarrow{\mathbf{p}}_{s}^{0}=\overrightarrow{\mathbf{a}}_{s}$, with $\overrightarrow{\mathbf{p}}_{s}^{0}=$ $\left(p(0), \ldots, p\left(W_{1}\right), 0, \ldots, 0\right)$, where $W_{1}$ is determined from the relation $\left\{0, \ldots, W_{1}\right\}=\{0, \ldots, W\} \cap S$.

The last equation can be presented as the system of equations

$$
\begin{gathered}
r_{0} p_{0}+r_{1} p_{1}+\ldots+r_{-W_{1}} p_{W_{1}}=a(0) ; \\
r_{1} p_{0}+r_{0} p_{0}+\ldots+r_{-W_{1}+1} p_{W_{1}}=a(1) ; \\
\ldots \\
r_{W_{1}} p_{0}+r_{W_{1}-1} p_{0}+\ldots+r_{0} p_{W_{1}}=a\left(W_{1}\right) ; \\
\ldots \\
r_{M_{s-1}+N_{s}} p_{0}+r_{1} p_{1}+\ldots+r_{0} p_{W_{1}}=a\left(M_{s-1}+N_{s}\right) .
\end{gathered}
$$

From the first $W_{1}$ equations we can find the unknown coefficients $p(k)$ and from the next equations we find the Fourier coefficients $r_{w}, w \in S \backslash\{0, \ldots, W\}$.

If the sequence $r_{w}, w \in S$, that is constructed from the strictly positive sequence $r_{w}, w \in S \cap\{0, \ldots, W\}$ and the calculated coefficients $r_{w}, w \in S \backslash\{0, \ldots, W\}$, is also strictly positive, then the least favourable spectral density $f_{0}(\lambda)$ is determined by the Fourier coefficients $r_{w}, w \in S$ of the function $f_{0}^{-1}(\lambda)$

$$
f_{0}(\lambda)=1 / \sum_{k=0}^{M_{s-1}+N_{s}}\left(r_{k} e^{i k \lambda}+r_{-k} e^{-i k \lambda}\right)=1 /\left|\sum_{k=0}^{M_{s-1}+N_{s}} \gamma_{k} e^{i k \lambda}\right|^{2} .
$$

Let us summarize our results and present them in the form of a theorem.

\section{Theorem 5.1}

The least favourable spectral density in the class $D_{W}$ for the optimal linear estimate of the functional $A_{s} \xi$ in the case where $W \geq M_{s-1}+N_{s}$ is the spectral density (20) of the autoregression stochastic sequence of order $W$ determined by coefficients $r_{w}, w=0,1, \ldots, W$. In the case where $W<M_{s-1}+N_{s}$ and solutions $r_{w}, w \in S \backslash\{0, \ldots, W\}$, to equation $B_{s} \overrightarrow{\mathbf{p}}_{s}^{0}=\overrightarrow{\mathbf{a}}_{s}$ together with coefficients $r_{w}, w \in S \cap\{0, \ldots, W\}$, form a strictly positive sequence, the least favourable spectral density in $D_{W}$ is the density (21) of the autoregression stochastic sequence of the order $M_{s-1}+N_{s}$. The minimax characteristic of the estimate is calculated by formula (11).

\section{Least favourable spectral densities in the class $D_{v}^{u}$}

Consider the problem of the optimal estimation of the functional $A_{s} \xi$ which depends on the unknown values of a stationary stochastic sequence $\xi(j)$ in the case where the spectral density of the sequence is from the set of spectral densities

$$
D_{u}^{v}=\left\{f(\lambda) \mid 0 \leq v(\lambda) \leq f(\lambda) \leq u(\lambda), \frac{1}{2 \pi} \int_{-\pi}^{\pi} f^{-1}(\lambda) d \lambda=P\right\},
$$

where $v(\lambda), u(\lambda)$ are given bounded spectral densities. Let the sequence $a(j), j \in S$, that determines the functional $A_{s} \xi$ be strictly positive. To find solutions to the constrained optimization problem (15) for the set $D_{u}^{v}$ of admissible spectral densities we use the condition $0 \in \partial \Delta_{D}\left(f_{0}\right)$. It follows from the condition $0 \in \partial \Delta_{D}\left(f_{0}\right)$ for $D=D_{u}^{v}$ that the Fourier coefficients of the function $f_{0}^{-1}$ satisfy both equation $B_{s}^{0} \overrightarrow{\mathbf{c}}_{s}=\overrightarrow{\mathbf{a}}_{s}$ and the equation

$$
\left|\sum_{l=0}^{s-1} \sum_{k=M_{l}}^{M_{l}+N_{l+1}}\left(\left(B_{s}^{0}\right)^{-1} \overrightarrow{\mathbf{a}}_{s}\right)_{k} e^{i k \lambda}\right|^{2}=\psi_{1}(\lambda)+\psi_{2}(\lambda)+p_{0}^{-2},
$$


where $\psi_{1}(\lambda) \geq 0$ and $\psi_{1}(\lambda)=0$ if $f_{0}(\lambda) \geq v(\lambda) ; \psi_{2}(\lambda) \leq 0$ and $\psi_{2}(\lambda)=0$ if $f_{0}(\lambda) \leq u(\lambda)$. Therefore, in the case where $v(\lambda) \leq f_{0}(\lambda) \leq u(\lambda)$, the function $f_{0}^{-1}(\lambda)$ is of the form

$$
f_{0}^{-1}(\lambda)=\sum_{k=0}^{M_{s-1}+N_{s}}\left(r_{k} e^{i k \lambda}+r_{-k} e^{-i k \lambda}\right)=\left|\sum_{k=0}^{M_{s-1}+N_{s}} \gamma_{k} e^{i k \lambda}\right|^{2}
$$

where $r_{k}=r_{-k}=P a(k) a^{-1}(0)$. The least favourable in the class $D_{u}^{v}$ is the density of the autoregression stochastic sequence of the order $M_{s-1}+N_{s}$ if the following inequality holds true

$$
v^{-1}(\lambda) \geq \sum_{k=0}^{M_{s-1}+N_{s}}\left(r_{k} e^{i k \lambda}+r_{-k} e^{-i k \lambda}\right)=\left|\sum_{k=0}^{M_{s-1}+N_{s}} \gamma_{k} e^{i k \lambda}\right|^{2} \geq u^{-1}(\lambda), \lambda \in[-\pi, \pi] .
$$

In general case the least favourable density is of the form

$$
f_{0}(\lambda)=\max \left\{v(\lambda), \min \left\{u(\lambda),\left|p_{0} \sum_{l=0}^{s-1} \sum_{k=M_{l}}^{M_{l}+N_{l+1}}\left(\left(B_{s}^{0}\right)^{-1} \overrightarrow{\mathbf{a}}_{s}\right)_{k} e^{i k \lambda}\right|^{2}\right\}\right\} .
$$

The following theorem holds true.

Theorem 6.1

If the sequence $a(j), j \in S$, is strictly positive and coefficients $r_{k}=r_{-k}=P a(k) a^{-1}(0), k \in S$, satisfy the inequality (22), then the least favourable in the class $D_{v}^{u}$ spectral density for the optimal linear estimate of the functional $A_{s} \xi$ is density (17) of the autoregression stochastic sequence of order $M_{s-1}+N_{s}$. The minimax characteristic $h\left(f_{0}\right)$ of the estimate can be calculated by the formula (18). If the inequality (22) is not satisfied, then the least favourable spectral density in $D_{v}^{u}$ is determined by relation (23) and the extremum condition (14). The minimax characteristic of the estimate is calculated by formula (11).

\section{Conclusions}

In this article we describe methods of solution of the problem of the mean-square optimal linear estimation of the functional $A_{s} \xi=\sum_{l=0}^{s-1} \sum_{j=M_{l}}^{M_{l}+N_{l+1}} a(j) \xi(j), M_{l}=\sum_{k=0}^{l}\left(N_{k}+K_{k}\right), N_{0}=K_{0}=0$, which depends on the unknown values of the stationary stochastic sequence $\xi(j)$. Estimates are based on observations of the sequence $\xi(j)$ at points $j \in \mathbb{Z} \backslash S$, where $S=\bigcup_{l=0}^{s-1}\left\{M_{l}, M_{l}+1, \ldots, M_{l}+N_{l+1}\right\}$. We provide formulas for calculating the values of the mean square error and the spectral characteristic of the optimal linear estimate of the functional in the case where the spectral density of the sequence $\xi(j)$ is exactly known. In the case where the spectral density is unknown, but a set of admissible spectral densities is given, the minimax approach is applied. We obtain formulas that determine the least favourable spectral densities and the minimax spectral characteristics of the optimal linear estimates of the functional $A_{s} \xi$ for concrete classes of admissible spectral densities. It is shown that spectral densities the autoregressive stochastic sequences are the least favourable in some classes of spectral densities.

\section{REFERENCES}

1. P. Bondon, Influence of missing values on the prediction of a stationary time series, Journal of Time Series Analysis, vol. 26, no. 4, pp. 519-525, 2005.

2. P. Bondon, Prediction with incomplete past of a stationary process, Stochastic Process and their Applications. vol.98, pp. 67-76, 2002.

3. I. I. Dubovets'ka, O.Yu. Masyutka, and M.P. Moklyachuk, Interpolation of periodically correlated stochastic sequences, Theory of Probability and Mathematical Statistics, vol. 84, pp. 43-56, 2012. 
4. I. I. Dubovets'ka, and M. P. Moklyachuk, Filtration of linear functionals of periodically correlated sequences, Theory of Probability and Mathematical Statistics, vol. 86, pp. 51-64, 2013.

5. I. I. Dubovets'ka, and M. P. Moklyachuk, Extrapolation of periodically correlated processes from observations with noise, Theory of Probability and Mathematical Statistics, vol. 88, pp. 43-55, 2013.

6. I. I. Dubovets'ka, and M. P. Moklyachuk, Minimaxestimation problem for periodically correlated stochastic processes, Journal of Mathematics and System Science, vol. 3, no. 1, pp. 26-30, 2013.

7. I. I. Dubovets'ka, and M. P. Moklyachuk, On minimax estimation problems for periodically correlated stochastic processes, Contemporary Mathematics and Statistics, vol.2, no. 1, pp. 123-150, 2014.

8. J. Franke, Minimax robust prediction of discrete time series, Z. Wahrscheinlichkeitstheor. Verw. Gebiete, vol. 68, pp. 337-364, 1985.

9. J. Franke and H. V. Poor, Minimax-robust filtering and finite-length robust predictors, Robust and Nonlinear Time Series Analysis. Lecture Notes in Statistics, Springer-Verlag, vol. 26, pp. 87-126, 1984.

10. I. I. Gikhman and A. V. Skorokhod, The theory of stochastic processes. I., Berlin: Springer, 2004.

11. I. I. Golichenko and M. P. Moklyachuk, Estimates of functionals of periodically correlated processes, Kyiv: NVP "Interservis", 2014.

12. U. Grenander, A prediction problem in game theory, Arkiv för Matematik, vol. 3, pp. 371-379, 1957.

13. E. J. Hannan, Multiple time series, Wiley Series in Probability and Mathematical Statistics. New York etc.: John Wiley \& Sons, Inc. XI, 1970.

14. A. D. Ioffe, and V. M. Tihomirov, Theory of extremal problems, Studies in Mathematics and its Applications, Vol. 6. Amsterdam, New York, Oxford: North-Holland Publishing Company. XII, 1979.

15. K. Karhunen, Über lineare Methoden in der Wahrscheinlichkeitsrechnung, Annales Academiae Scientiarum Fennicae. Ser. A I, vol. 37, 1947.

16. Y. Kasahara, and M. Pourahmadi, and A. Inoue, Duals of random vectors and processes with applications to prediction problems with missing values, Statistics \& Probability Letters, vol. 79, no. 14, pp. 1637-1646, 2009.

17. S. A. Kassam and H. V. Poor, Robust techniques for signal processing: A survey, Proceedings of the IEEE, vol. 73, no. 3, pp. 433-481, 1985.

18. A. N. Kolmogorov, Selected works by A. N. Kolmogorov. Vol. II: Probability theory and mathematical statistics. Ed. by A. N. Shiryayev, Mathematics and Its Applications. Soviet Series. 26. Dordrecht etc. Kluwer Academic Publishers, 1992.

19. M. G. Krein and A. A. Nudelman, The Markov moment problem and extremal problems, Translations of Mathematical Monographs. Vol. 50. Providence, R.I.: American Mathematical Society, 1977.

20. M. M. Luz and M. P. Moklyachuk, Interpolation of functionals of stochastic sequences with stationary increments, Theory of Probability and Mathematical Statistics, vol. 87, pp. 117-133, 2012.

21. M. M. Luz, and M. P. Moklyachuk, Minimax-robust filtering problem for stochastic sequence with stationary increments, Theory of Probability and Mathematical Statistics, vol. 89, pp. 127 - 142, 2014.

22. M. Luz, and M. Moklyachuk, Minimax-robust filtering problem for stochastic sequences with stationary increments and cointegrated sequences, cStatistics, Optimization \& Information Computing, vol. 2, no. 3, pp. 176 - 199, 2014.

23. M. Luz, and M. Moklyachuk, Minimax interpolation problem for random processes with stationary increments, Statistics, Optimization \& Information Computing, vol. 3, no. 1, pp. 30-41, 2015.

24. M. Luz, and M. Moklyachuk, Minimax-robust prediction problem for stochastic sequences with stationary increments and cointegrated sequences, Statistics, Optimization \& Information Computing, vol. 3, no. 2, pp. 160-188, 2015.

25. M. P. Moklyachuk, Minimax extrapolation and autoregressive-moving average processes, Theory of Probability and Mathematical Statistics, vol. 41, pp. 77-84, 1990.

26. M. P. Moklyachuk, Stochastic autoregressive sequences and minimax interpolation, Theory of Probability and Mathematical Statistics, vol. 48, pp. 95-103, 1994.

27. M. P. Moklyachuk, Robust procedures in time series analysis, Theory of Stochastic Processes, vol. 6, no. 3-4, pp. 127-147, 2000.

28. M. P. Moklyachuk, Game theory and convex optimization methods in robust estimation problems, Theory of Stochastic Processes, vol. 7, no. 1-2, pp. 253-264, 2001.

29. M. P. Moklyachuk, Robust estimations of functionals of stochastic processes, Kyiv University, Kyiv, 2008.

30. M. Moklyachuk and O. Masyutka, Minimax-robust estimation technique for stationary stochastic processes, LAP LAMBERT Academic Publishing, 2012.

31. M. Pourahmadi, A. Inoue and Y. Kasahara A prediction problem in $L^{2}(w)$. Proceedings of the American Mathematical Society. Vol. 135, No. 4, pp. 1233-1239, 2007.

32. B. N. Pshenichnyj, Necessary conditions of an extremum, Pure and Applied mathematics. 4. New York: Marcel Dekker, 1971.

33. R. T. Rockafellar, Convex Analysis, Princeton University Press, 1997.

34. Yu. A. Rozanov, Stationary stochastic processes, San Francisco-Cambridge-London-Amsterdam: Holden-Day, 1967.

35. H. Salehi, Algorithms for linear interpolator and interpolation error for minimal stationary stochastic processes, The Annals of Probability, Vol. 7, No. 5, pp. 840-846, 1979.

36. K. S. Vastola and H. V. Poor, An analysis of the effects of spectral uncertainty on Wiener filtering, Automatica, vol. 28, pp. 289-293, 1983.

37. N. Wiener, Extrapolation, interpolation and smoothing of stationary time series. With engineering applications, The M. I. T. Press, Massachusetts Institute of Technology, Cambridge, Mass., 1966.

38. A. M. Yaglom, Correlation theory of stationary and related random functions. Vol. 1: Basic results, Springer Series in Statistics, Springer-Verlag, New York etc., 1987.

39. A. M. Yaglom, Correlation theory of stationary and related random functions. Vol. 2: Supplementary notes and references, Springer Series in Statistics, Springer-Verlag, New York etc., 1987. 